\title{
New insights for early assessment of cardiac involvement in Anderson-Fabry disease
}

\author{
Alberto Cuocolo, MD, a Carmela Nappi, MD, ${ }^{\text {a }}$ Valeria Gaudieri, MD, PhD, ${ }^{\text {a }}$ \\ Antonio Pisani, $\mathrm{MD}, \mathrm{PhD},^{\mathrm{b}}$ and Massimo Imbriaco, $\mathrm{MD}^{\mathrm{a}}$ \\ a Department of Advanced Biomedical Sciences, University "Federico II", Naples, Italy \\ b Department of Public Health, University "Federico II", Naples, Italy
}

Received Jan 23, 2019; accepted Jan 23, 2019

doi: $10.1007 / \mathrm{s} 12350-019-01635-w$

Anderson-Fabry disease (AFD) is an X-linked recessive lysosomal storage disorder that is caused by the deficient activity of a-galactosidase A (a-Gal A) ${ }^{1}$ with the resultant accumulation of globotriaosylceramide (Gb3) and related glycosphingolipids ${ }^{2}$ in a variety of cells, including micro-vascular endothelial cells, renal tubular cells, and myocardial cells. In classically affected males, the progressive Gb3 accumulation leads to renal, cardiac, and cerebro-vascular manifestations and early death. ${ }^{3}$ Chronic renal failure represents the most frequent cause of morbidity, with cardiac involvement being the leading cause of death and premature mortality. ${ }^{4}$ It has been reported that the progressive accumulation of Gb3 in cardiomyocytes, cardiac valves, endothelial cells, and conduction system, leads to increased ventricular wall thickness and functional impairment, ${ }^{5}$ in addition to valvular and electrocardiographic abnormalities. ${ }^{6}$ The classic cardiac involvement is a form of hypertrophic cardiomyopathy, usually described as concentric left ventricular hypertrophy (LVH). The disease is progressive, with symptoms appearing with increasing age.

Myocardial fibrosis is generally considered a sign of disease progression, although it may also be present in female patients without LVH, suggesting that hypertrophy and fibrosis are not necessarily associated. ${ }^{7}$ Although the diagnosis of AFD is based on enzyme assay and genetic testing, there is an increasing need to identify potential phenotypic markers that can detect early cardiac involvement, preferably before the development of myocardial damage and fibrosis and potentially determine treatment response. Indeed, there

\footnotetext{
Reprint requests: Alberto Cuocolo, MD, Department of Advanced Biomedical Sciences, University Federico II, Via Pansini 5, 80131 Naples, Italy; cuocolo@unina.it

J Nucl Cardiol 2021;28:2500-2.

$1071-3581 / \$ 34.00$

Copyright (C) 2019 American Society of Nuclear Cardiology.
}

is agreement that early enzyme replacement therapy in pre-hypertrophic Fabry cardiomyopathy, prevents progression of the disease, strongly influencing life expectancy.

Conventional two-dimensional echocardiography is the standard imaging tool for identifying cardiac involvement in AFD but is not suitable to detect subtle myocardial dysfunction in the early course of the disease. Advanced echocardiographic techniques, including longitudinal strain, using speckle-tracking echocardiography, have recently been proposed as sensitive markers of myocardial deformation in patients with AFD, independently of the presence of $\mathrm{LVH}$, as well in naïve AFD patients, mainly involving LV basal myocardial segments. ${ }^{8}$

Cardiac magnetic resonance (CMR) offers the unique possibility of measuring LV mass, dimensions, and wall thickness, together with a non-invasive tissue characterization for assessment of replacement fibrosis or inflammation. It has been largely demonstrated the usefulness of late-gadolinium enhancement (LGE) CMR technique, in the recognition of myocardial fibrosis in the setting of cardiac involvement of AFD disease. ${ }^{9-12}$ LGE occurs in up to $64 \%$ of patients with AFD, with the majority displaying LGE localized to the basal inferolateral wall. ${ }^{13-16}$ Previous reports have also suggested that LGE in the context of normal mass is a phenomenon observed predominantly in women. ${ }^{17} \mathrm{~T} 1$ mapping is an additional and powerful diagnostic tool in AFD, that has been recently introduced in the diagnostic work up of these patients, to discriminate AFD from other causes of $\mathrm{LVH}^{18}{ }^{18}$ Pica et al. ${ }^{18}$ demonstrated that even in subjects without LVH, reduced myocardial T1 has a high prevalence and is associated with echocardiographic parameters of LV dysfunction, suggesting that a low T1 is detecting pre-hypertrophic or early cardiac involvement in AFD, as a consequence of the progressive Gb3 
storage in the myocardium ${ }^{19}$ and may be a starting criterion for early treatment.

Novel concepts that consider alternative molecular pathways linking Gb3 storage and fibrosis and integrated multimodality imaging using PET-MR acquisition have been shown to provide at the same time information on intra-myocardial fibrosis and areas of active myocardial inflammation in patients with $\mathrm{AFD}{ }^{20}$ In particular, Spinelli et al. ${ }^{21}$ recently demonstrated in a prospective comparative study, using PET-MR and two-dimensional strain echocardiography, in 24 heterozygous females carrying $\alpha$-Gal A mutation and without $\mathrm{LVH}$, that focal FDG uptake represents an early sign of disease-related myocardial damage and is associated to LV longitudinal function impairment. These findings support the hypothesis that inflammation is likely to represent a clue of myocardial response to Gb3 storage and may be the pathological link in phenotype development of the disease. Further supporting these data, Frustaci et al. ${ }^{22}$ reported that $\mathrm{Gb} 3$ accumulation in Fabry cells, generate a pro-inflammatory response by the human immune system and found detectable myocarditis at histology in $56 \%$ of patients with AFD.

Another challenging field of research has also been recently proposed using ${ }^{123}$ I-metaiodobenzylguanidine $\left({ }^{123} \mathrm{I}-\mathrm{MIBG}\right)$ myocardial scintigraphy that allows to measure post-ganglionic pre-synaptic noradrenergic uptake in vivo and can therefore provide information on the sympathetic activity and its correlation with $\mathrm{LV}$ diastolic dysfunction. ${ }^{23,24}$ In particular, Imbriaco et al. ${ }^{25}$ demonstrated that in patients with AFD there is reduced ${ }^{123}$ I-MIBG uptake in the LV myocardium compared to controls; this reduced ${ }^{123} \mathrm{I}$-MIBG uptake is significantly higher in the infero-lateral region, compared to the remaining myocardial walls and is also present in patients without evidence of myocardial fibrosis, as assessed on CMR. The authors showed that in $50 \%$ of patients with no evidence of myocardial fibrosis on LGE-CMR, a regional reduction of ${ }^{123}$ I-MIBG uptake was observed in the infero-lateral wall, suggesting that degenerative changes in cardiac sympathetic nerves may precede the onset of myocardial fibrosis. ${ }^{25}$ These data suggest that cardiac adrenergic denervation due to autonomic nervous degeneration accounts for alterations in ${ }^{123}$ I-MIBG myocardial imaging and the impaired sympathetic function observed in patients with AFD.

In conclusion, although two-dimensional echocardiography remains the primary tool for initial evaluation and follow-up of patients with AFD, more advanced techniques such as CMR with T1-mapping and speckle tracking echocardiography can be particularly useful especially in patients with severe renal impairment, for whom intravenous contrast is contraindicated and as initial investigation of patients with $\alpha$-Gal A mutation.
Further studies are mandatory to definitively establish the effectiveness of nuclear medicine techniques such as ${ }^{123}$ I-MIBG myocardial scintigraphy and integrated multimodality PET-MR imaging, to assess early myocardial involvement in AFD.

\section{Disclosure}

A. Cuocolo, C. Nappi, V. Gaudieri, A. Pisani, M. Imbriaco declare that they have no conflict of interest.

\section{References}

1. Desnick RJ, Ioannou YA, Eng CM. $\alpha$-Galactosidase A deficiency: Fabry disease. In: Scriver CR, Beaudet AL, Sly WS, Valle D, editors. The metabolic and molecular bases of inherited disease. 8th ed. New York: McGraw Hill; 2001. p. 3733-74.

2. Germain DP. Fabry disease. Orphanet J Rare Dis. 2010;5:30.

3. Linhart A, Kampmann C, Zamorano JL, Sunder-Plassmann G, Beck M, Mehta A, et al. European FOS investigators. Cardiac manifestations of Anderson-Fabry disease: results from the international Fabry outcome survey. Eur Heart J. 2007;28:1228-35.

4. Pisani A, Visciano B, Imbriaco M, Di Nuzzi A, Mancini A, Marchetiello C, et al. The kidney in Fabry's disease. Clin Genet. 2014;86:301-9.

5. Spinelli L, Nicolai E, Acampa W, Imbriaco M, Pisani A, Rao MA, et al. Cardiac performance during exercise in patients with Fabry's disease. Eur J Clin Invest. 2008;38:910-7.

6. Kozor R, Grieve SM, Tchan MC, Callaghan F, Hamilton-Craig C, Denaro $\mathrm{C}$, et al. Cardiac involvement in genotype-positive Fabry disease patients assessed by cardiovascular MR. Heart. 2016;102:298-302.

7. Weidemann F, Sanchez-Niño MD, Politei J, Oliveira JP, Wanner C, Warnock DG, et al. Fibrosis: a key feature of Fabry disease with potential therapeutic implications. Orphanet J Rare Dis. 2013;8:116.

8. Krämer J, Niemann M, Liu D, Hu K, MacHann W, Beer M, et al. Two-dimensional speckle tracking as a non-invasive tool for identification of myocardial fibrosis in Fabry disease. Eur Heart J. 2013;34:1587-96.

9. Imbriaco M, Spinelli L, Cuocolo A, Maurea S, Sica G, Quarantelli $\mathrm{M}$, et al. MRI characterization of myocardial tissue in patients with Fabry's disease. AJR Am J Roentgenol. 2007;188:850-3.

10. Imbriaco M, Pisani A, Spinelli L, Cuocolo A, Messalli G, Capuano E, et al. Effects of enzyme-replacement therapy in patients with Anderson-Fabry disease: a prospective long-term cardiac magnetic resonance imaging study. Heart. 2009;95:1103-7.

11. Imbriaco M, Messalli G, Avitabile G, Cuocolo A, Maurea S, Soscia F, et al. Cardiac magnetic resonance imaging illustrating Anderson-Fabry disease progression. Br J Radiol. 2010;83:e24951.

12. Moon JC, Sachdev B, Elkington AG, McKenna WJ, Mehta A, Pennell DJ, et al. Gadolinium enhanced cardiovascular magnetic resonance in Anderson-Fabry disease. Evidence for a disease specific abnormality of the myocardial interstitium. Eur Heart $\mathbf{J}$ 2003;24:2151-5.

13. Moon JC, Sheppard M, Reed E, et al. The histological basis of late gadolinium enhancement cardiovascular magnetic resonance in a patient with Anderson-Fabry disease. J Cardiovasc Magn Reson. 2006;8:479-82. 
14. Mewton N, Liu CY, Croisille P, Bluemke D, Lima JA. Assessment of myocardial fibrosis with cardiovascular magnetic resonance. J Am Coll Cardiol. 2011;57:891-903.

15. Wu KC, Weiss RG, Thiemann DR, Kitagawa K, Schmidt A, Dalal $\mathrm{D}$, et al. Late gadolinium enhancement by cardiovascular magnetic resonance heralds an adverse prognosis in nonischemic cardiomyopathy. J Am Coll Cardiol. 2008;51:2414-21.

16. Stirrat J, White JA. The prognostic role of late gadolinium enhancement magnetic resonance imaging in patients with cardiomyopathy. Can J Cardiol. 2013;29:329-36.

17. Niemann M, Herrmann S, Hu K, Breunig F, Strotmann J, Beer M, et al. Differences in Fabry cardiomyopathy between female and male patients: Consequences for diagnostic assessment. JACC Cardiovasc Imaging. 2011;4:592-601.

18. Pica S, Sado DM, Maestrini V, Fontana M, White SK, Treibel T, et al. Reproducibility of native myocardial $\mathrm{T} 1$ mapping in the assessment of Fabry disease and its role in early detection of cardiac involvement by cardiovascular magnetic resonance. J Cardiovasc Magn Reson. 2014;5(16):99.

19. Sado DM, White SK, Piechnik SK, Banypersad SM, Treibel T, Captur G, et al. Identification and assessment of Anderson-Fabry disease by cardiovascular magnetic resonance non-contrast myocardial T1 mapping. Circ Cardiovasc Imaging. 2013;6:392-8.

20. Nappi C, Altiero M, Imbriaco M, Nicolai E, Giudice CA, Aiello $\mathrm{M}$, et al. First experience of simultaneous PET/MRI for the early detection of cardiac involvement in patients with Anderson-Fabry disease. Eur J Nucl Med Mol Imaging. 2015;42:1025-31.
21. Spinelli L, Imbriaco M, Nappi C, Nicolai E, Giugliano G, Ponsiglione A, et al. Early cardiac involvement affects left ventricular longitudinal function in females carrying $\alpha$-galactosidase $\mathrm{A}$ mutation: Role of hybrid positron emission tomography and magnetic resonance imaging and speckle-tracking echocardiography. Circ Cardiovasc Imaging. 2018;11:e007019.

22. Frustaci A, Verardo R, Grande C, Galea N, Piselli P, Carbone I, et al. Immune-mediates myocarditis in Fabry disease cardiomyopathy. J Am Heart Assoc. 2018;7:e009052.

23. Verschure DO, Lutter R, van Eck-Smit BL, Somsen GA, Verberne HJ. Myocardial 123I-MIBG scintigraphy in relation to markers of inflammation and long-term clinical outcome in patients with stable chronic heart failure. J Nucl Cardiol. 2018;25:845-53.

24. Spinelli L, Pellegrino T, Pisani A, Giudice CA, Riccio E, Imbriaco $\mathrm{M}$, et al. Relationship between left ventricular diastolic function and myocardial sympathetic denervation measured by (123) Imeta-iodobenzylguanidine imaging in Anderson-Fabry disease. Eur J Nucl Med Mol Imaging. 2016;43:729-39.

25. Imbriaco M, Pellegrino T, Piscopo V, Petretta M, Ponsiglione A, Nappi C, et al. Cardiac sympathetic neuronal damage precedes myocardial fibrosis in patients with Anderson-Fabry disease. Eur J Nucl Med Mol Imaging. 2017;44:2266-73.

Publisher's Note Springer Nature remains neutral with regard to jurisdictional claims in published maps and institutional affiliations. 